\title{
Continuous Casting Research in the USSR
}

\author{
Abstracted from the British \\ Iron and Coal Trades Review
}

\begin{abstract}
I Russia, the most important theoretical question 1 in the continuous casting of steel is considered to be the relationship between the depth and extent of the liquid phase in the continuously cast ingot, because the minimum distance to the cut-off point is determined by this factor.
\end{abstract}

\section{Crystallization and liquid phase studies}

At the Novo-Tulskii Metallurgical Works, some experimental work was carried out to determine the depth of the liquid phase, the solidification boundary, called the crystallization front, and the speed of crystallization. Two methods were used: a) temperature measurements inside the solidifying continuous cast ingot; b) introduction of the $\mathrm{P}^{32}$ or the $\mathrm{S}^{32}$ isotope and lead into the ingot.

Measurement of the temperature inside the solidifying ingot was used for the determination of the duration of complete crystallization and for the determination of the average rate of crystallization. One thermocouple was placed in the center of the cross-sectional area of the ingot and another at 10 $\mathrm{mm}$ beneath the surface of the ingot. The readings of the central thermocouple were taken to determine the duration of complete crystallization, which corresponded to a kink in the temperature curve. The tests showed that, for an ingot 6 in. thick, the time for complete crystallization is $6 \mathrm{~min}$ and does not depend on the speed of casting. Consequently, for a given section, the depth of the liquid phase is determined by the expression $L=6 v$, in which $v$ is the linear speed of casting in $\mathrm{mm}$ per min.

To determine the depth of the liquid phase in the ingot, radioactive phosphorus $\mathrm{P}^{32}$ was mixed with normal elementary phosphorus and iron powder. The mixture was divided into five portions which were placed in a brass container fixed to the end of a steel rod $10 \mathrm{in.}$ long. The rod containing the radioactive phosphorus was inserted into the continuous-cast ingot at the end of pouring. The liquid phase of the ingot underwent rapid inoculation and thus the boundaries of the liquid phase were fixed. Longitudinal and cross-sectional templates were cut from the ingot and contact radiograms were taken from them so as to visualize the boundaries between the solid and liquid phases at the moment of introduction of the isotope.

According to the results of the investigations, the depth of the liquid phase should be: at the casting speed of $315 \mathrm{~mm}$ per $\min , 315 \times 6=1890 \mathrm{~mm}$ and with a casting speed of $700 \mathrm{~mm}$ per min, $700 \times 6=$ $4200 \mathrm{~mm}$. Thus the results of both methods are in agreement and confirm that the depth of the liquid phase in a continuous-cast ingot is directly proportional to the speed of casting. The depth of the liquid phase, as calculated by Roth's formula is much too high.

\section{Zone of contact between ingot skin and mold wall}

'The skin is in contact with the walls of the mold only for a small distance, the skin separating from the walls because of shrinkage, forming a gas gap. In order to determine rational dimensions of a mold, it is necessary to know the extent of the zone of contact and the size of the shrinkage gap.

The extent of the zone of contact between ingot and mold was determined according to the temperature of the inner surface of the brass wall. To do this, riveted or cold-hammered brass plates were placed in the joint between the wide and narrow plates of the mold throughout its entire length and after the steel was poured, the hardness of these plates was measured. The part with the minimum hardness corresponded to the maximum temperature of heating. An investigation carried out in this way showed that the zone of contact on pouring at a speed of about $16 \mathrm{ft}$ per min was $51 / 2$ to 6 in. at the narrow walls.

Similar results were obtained in subsequent experiments in which temperature of the brass walls was measured by thermocouples. A calculation of the heat loss according to temperatures along the height of the narrow walls of the mold indicates a sudden decrease in heat extraction at the moment the skin parts from the walls. The middle of the wide face of the ingot parts from the walls of the mold somewhat later, because it is buckled under the action of ferrostatic pressure.

\section{Conclusions}

From these and other studies, it is concluded that:

1) Under experimental conditions, the depth of the liquid phase in the continuously cast ingot is directly proportional to the speed of extraction of the ingot.

2) In connection with the difference in thickness of the solidifying skin at the perimeter of a rectangular ingot, it is essential to modify the design of the mold so as to obtain a uniform heat extraction throughout the entire perimeter of the ingot.

3) The zone of contact between the solidifying skin of the continuously cast ingot of carbon steel and the narrow wall of the mold extends from $5 \frac{1 / 2}{2}$ to $71 / 4 \mathrm{in}$. and is dependent upon the casting speed.

4) An increase in the speed of continuous casting causes a decrease in the amount of heat extraction from the ingot both in the mold and in the zone of secondary cooling.

5) An increase in the water consumption for secondary cooling of more than 6 liters per $\mathrm{kg}$ of steel does not increase the intensity of cooling of the ingot. 